\title{
Liver Involvement in Systemic Lupus Erythematosus Patients
}

\author{
Amira A. Shahin, Ghada S. Alazkalany, Maha S. Hasaballah, and \\ Al Shaymaa A. Ewiela*
}

Department of Rheumatology \& Rehabilitation, Faculty of Medicine, Cairo university, Egypt

\begin{abstract}
Background: Systemic lupus erythematosus (SLE) is a potentially severe, frequently disabling autoimmune disease with multi-organ involvement. Liver involvement in SLE is not uncommon. It is frequently asymptomatic and limited to liver tests abnormalities. Aim: To detect the frequency and nature of liver involvement in SLE patients and to correlate these with other organ involvement. Patients and Methods: A retrospective study included 200 SLE patients who had admitted at Rheumatology and Rehabilitation department, Faculty of Medicine, Cairo university. Patients were classified into two groups Group I (no liver affection) and Group II (with liver affection). These groups were subdivided into subgroups; A (6 patients with AIH), B (194 patients without AIH), C (13 patients with fatty changes), D ( 187 patients without fatty changes), E ( 6 patients with HCV) \& F (194 patients without HCV). Patients were subjected to full history taking, clinical examination and laboratory investigations; the erythrocyte sedimentation rate (ESR), complete blood count (CBC), serum creatinine, alanine transaminases (ALT), aspartate transaminases (AST), Autoantibodies (ANA and Anti DNA, and Anti LKM), serum complement levels $\left(\mathrm{C}_{3}, \mathrm{C}_{4}\right)$, urine analysis, total albumin in 24 hours urine and HCV by PCR. Liver and renal biopsies were done in selected patients. Patients were also subjected to plain X-ray and abdominal sonography. Assessment of disease damage was measured by using systemic lupus erythematosus international collaborating clinic (SLICC). Results: Liver involvement was found in 23 SLE patients (11\%). We classified the 23 patients into 5 major groups: Autoimmune hepatitis in 6 patients (26\%), Cholangitis in 1 patient (4.3\%), portal venous thrombosis in 2 patients $(8.6 \%)$, Cirrhosis in 1 patient (4.3\%), Liver congestion in 1 patient (4.3\%), fatty changes in 13 patients (56.6\%), HCV in 6 patients (26\%). A significant relation between the age of onset, presence of ascites and SLICC in group $A$ compared to $B(p=0.034, p=0.007$ and $p=0.010$ respectively) was found. Conclusion: Liver in SLE is the least organ affected. The younger the age group of lupus patients with $\mathrm{AlH}$, the more the organ damage.
\end{abstract}

Keywords: AlH, nodular regenerative hyperplasia, Antiphospholipid syndrome

\section{Introduction}

Systemic lupus erythmatosus (SLE) is a potentially severe, frequently disabling autoimmune disease with multi-organ in- volvement and a typically waxing and waning course ${ }^{(1)}$. SLE often harms the heart, joints, lung, blood vessels, liver, kidneys, and nervous system(2). Liver involvement in SLE is not uncommon. It is 
frequently asymptomatic and limited to liver tests abnormalities(3). Patients with SLE have a $25-50 \%$ chance of developing abnormal liver tests in their life time ${ }^{(4)}$. Liver diseases in SLE include: hepatic congestion, fatty liver, arteritis, cholestasis, peliosis hepatic (numerous small bloodfilled cystic lesions throughout the liver), chronic persistent hepatitis, nonspecific reactive hepatitis, cholangitis (inflammation of the small bile capillaries), nodular regenerative hyperplasia of the liver (NRH), and hemangioma(5). Autoimmune liver disease (AILD) in patients with SLE, include: autoimmune hepatitis (AIH), primary biliary cirrhosis (BPC), and the $\mathrm{AlH}$ / PBC overlap syndrome ${ }^{(6)}$. Nodular regenerative hyperplasia ( $\mathrm{NRH}$ ) of liver is uncommon condition, it presents primarily with manifestations of portal hypertension(7). The main cause of liver involvement in SLE patients is previous treatment with hepatotoxic drugs or hepatotropic viral hepatitis ${ }^{(8)}$. SLE itself or treated with steroids does not seem to worsen the liver condition in patients with hepatitis $C$ virus (HCV) infection ${ }^{(9)}$. Severe liver involvement requiring liver transplantation in SLE, but very few cases have been reported. The patient persisted without reactivation of SLE and with long-term survival( $^{(10)}$.

\section{Patients and Methods}

Two hundred patients of SLE were included in this study from Rheumatology and Rehabilitation Department in Al-Kasr Al-Aini hospitals during the period starting from 1 January 2013 till January 2016. Full history taking and thorough clinical examination including details about liver is included in this study. They fulfilled the criteria of SLE according to American College of Rheumatology (ACR) criteria(11). All laboratory investigations include autoantibodies related to liver disease and radiological investigations was collected and analyzed. Even liver biopsies also were collected.

\section{Statistical analysis}

These data were collected, tabulated and analyzed by SPSS package version 10 (SPSS corporation USA), Qualitative data were presented in the form of number and percent and quantitative data were presented in the form of mean and standard deviation. Correlation between variables was performed using Regression Analysis (Pearson's correlation test). The kappa coefficient was used to describe agreement between both imaging techniques evaluated in this study.

\section{Results}

Liver involvement in SLE patients: Twentythree SLE patients (11.5\%) had liver involvement; autoimmune hepatitis in 6 (26\%), cholestatic jaundice in 1 patient (4.3\%), portal thrombosis in $2(8.6 \%)$, cirrhosis in 1 patient (4.3\%), Liver congestion in 1 patient (4.3\%), fatty changes in liver in 13 (56.6\%), and viral hepatitis in the form of HCV in 6 (26\%) (Figure 1).

Clinical presentation: The patients examined abdominally and showed: ascites in 5 (21.7\%), lower limb edema in 12 (52.1\%), jaundice in $5(21.7 \%)$, abdominal tenderness in 20 (86.6\%), hepatomegaly in 14 (60.8\%), and splenomegaly in 7 (30.4\%). Sonographic examination: In our collected 200 patients the 23 patients associated with liver involvement were investigated sonographically by abdominal ultrasound which showed fatty fatty changes in (56.6\%), hepatomegaly in (54.5\%), splenomegaly in (34.5\%) and congested liver in one patient (4.3\%). By using Doppler, we detected 2 patients having portal venous thrombosis. 
Liver biopsy: Only 6 of our 23 SLE patients associated with liver involvement exposed to liver biopsy to confirm the diagnosis with AlH.

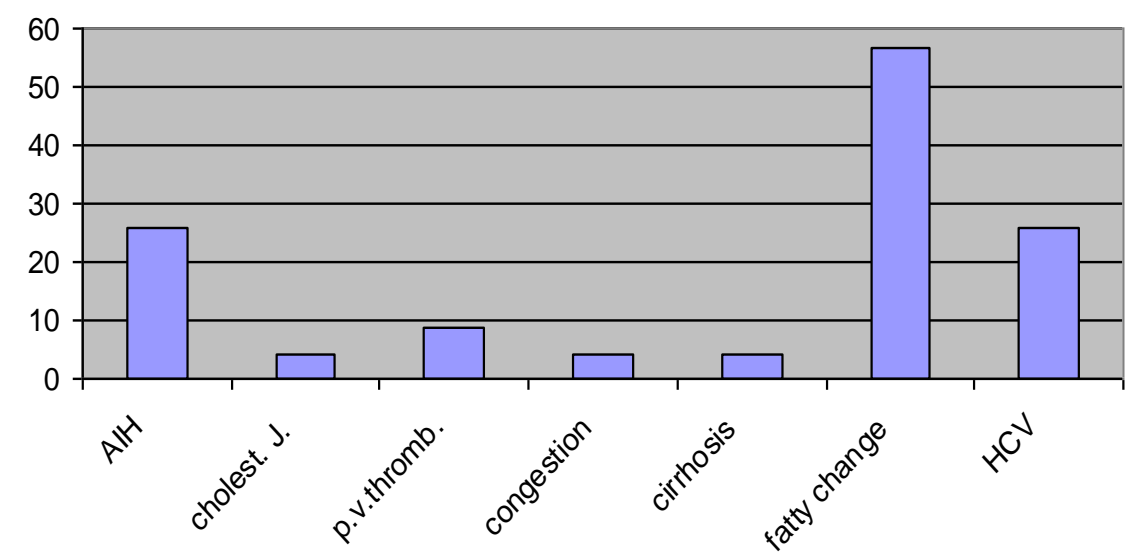

Figure 1: liver involvement in SLE patients (\%) AlH: autoimmune hepatitis; cholest. J: cholestatic jaundice; p.v.thromb: portal venous thrombosis; $\mathrm{HCV}$ :hepatitis $\mathrm{C}$ virus

Autoimmune hepatitis: We divided our 200 SLE patients into 2 groups: group (A) which included 6 patients with $\mathrm{AlH}$ and group (B) which included 194 patients without $\mathrm{AlH}$. The mean age of onset of lupus in group A was significantly higher than the mean age of onset in group $B$ (14.2 \pm 5.4 vs. $21.4 \pm 8.2$ yrs, $p=0.034)$. The mean SLICC in group A was significantly higher than that in group $B(3.5 \pm 1.5 \mathrm{vs}$.
1.9 $\pm 1.5, p=0.010$ (Table 2).

Cholestatic jaundice: One female patient had cholestatic jaundice. She was 30 years old, age of onset of the disease was 25 years old (i.e. 5 years disease duration). Portal venous thrombosis: Considering two female patients having portal thrombosis we found that their ages were 27\&25 years old, age of onset were $20 \& 16$ years old and disease duration was $7 \& 9$ years.

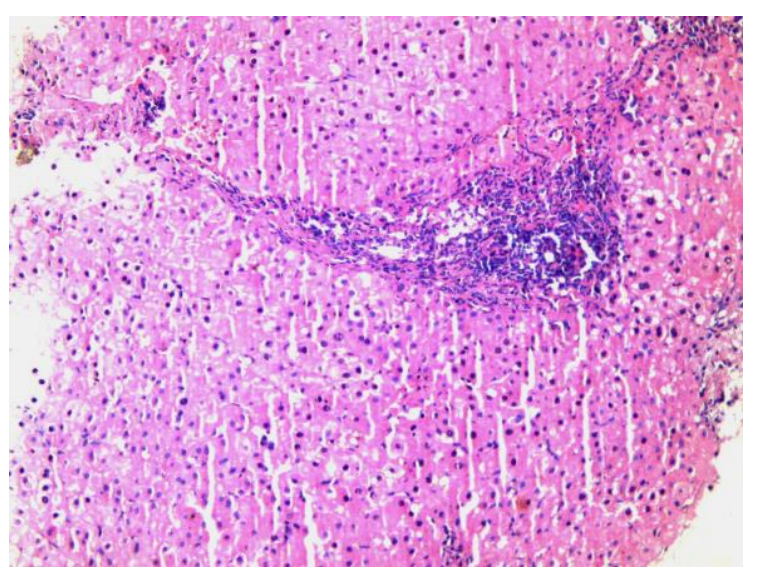

Figure 2: AlH histopathology

Fatty changes: We divided our 200 SLE patients into 13 patients with fatty changes in liver (group C) and 187 patients without fatty changes (group D). By comparing the mean SLICC in both groups C and D, we found that the mean SLICC in in group $C$ was insignificantly higher than the mean SLICC in group $D(2.1 \pm 1.2,2.9 \pm 1.8, p=0.46)$. 
Liver Cirrhosis: Only one female patient (27 years old) had liver cirrhosis, her age of onset was 20 years old and the disease duration was 7 years.

Liver congestion: One female patient (27 years old) had liver congestion, age of onset of the disease was 18 years old and disease duration was 9 years.
Viral Hepatitis in the form of HCV: We classified our 200 SLE patients into 6 patients with viral hepatitis in the form of HCV (group E), and 194 patients without viral hepatitis (group F). By comparing the mean SLICC in both groups $E$ and $F$, no significant relation between both $(2.0 \pm 1.0$ vs. $3.1 \pm 1.0$ years $)(p=0.14)$ was found

Table 1: Comparison between demographic data in group A, and group $B$

\begin{tabular}{|l|c|c|c|}
\hline & A & $B$ & $P$ \\
\hline Mean age \pm SD (years) & $23.0 \pm 2.6$ & $27.8 \pm 7.7$ & 0.130 \\
\hline Mean age of onset \pm SD (years) & $14.2 \pm 5.4$ & $21.4 \pm 8.2$ & $0.034^{*}$ \\
\hline Mean disease duration \pm SD (years) & $8.8 \pm 4.5$ & $6.4 \pm 3.9$ & 0.145 \\
\hline
\end{tabular}

Table 2: Comparison between SLICC in group A, and B

\begin{tabular}{|c|c|c|c|}
\hline & A & B & P \\
\hline Mean SLICC \pm SD & $3.5 \pm 1.5$ & $1.9 \pm 1.5$ & $0.010^{*}$ \\
\hline
\end{tabular}

SLICC = systemic lupus erythematosus international collaborating clinic

\section{Discussion}

Systemic lupus erythematosus (SLE) is a systemic autoimmune disease that can affect any part of the body, the immune system attacks the body's cells and tissues, resulting in inflammation and tissue damage ${ }^{(12)}$. End-stage hepatic disease, namely development of portal hypertension, cirrhosis, and hepatic encephalopathy occurs rarely in patients with SLE ${ }^{(13)}$. There are several case reports and reviews on liver disease in SLE ${ }^{(14)}$. SLE most often harms the heart, joints, skin, lungs, blood vessels, kidneys, and nervous systems $^{(2)}$. About the presenting manifestations of SLE in 200 patients, the most common was the musculo-skeletal manifestations (arthralgia and arthritis) in 182 patients (91\%), followed by hematologic manifestations in 123 patients (61.5\%), followed by mucocutaneous manifestations in 122 patients (61\%). Then the lung disease presented in 115 patients (57.55\%) followed by renal disease in 108 patients (54\%) then CNS affection in 100 patients (50\%) followed by cardiac manifestations in 28 patients (14.5\%), followed by hematological disease in 13 patients (26\%), followed by eye lesions in 28 patients (14\%) and the least presentation was liver involvement in only 23 patients (11.5\%). If we compared our study with a similar study done by Chowdhary on 40 SLE patients it also found that the liver was the least organ affected in SLE which is like our results. And comparing the major organs involved in that study concerning the major organs involved they found that musculo-skeletal involvement is the $1^{\text {st }}$ major organ affected in (85\%) which is like our findings that it is $1^{\text {st }}$ presenting symptoms in $91 \%$ of our cases ${ }^{(15)}$. Also, mucocutaneous involvement in (82\%) of their patients, renal involvement in (50\%) and the lung in (49\%) of their cases. These cases relatively like our cases. But in their study both CNS \& Hematological involvement were found in (35\%) of cases, while in our study CNS involvement was $50 \%$ and hematologic manifestations were $61.5 \%$ of our studied cases. If we compared the serological features they found in their patients, we found that, in their study ANA was +ve in 
(85\%) of their patients, and Anti-DNA was +ve in (92.5\%) in their patients ${ }^{(15)}$. While in our study ANA was +ve in $99.5 \%$ of our patients, and Anti dsDNA was +ve in $53.3 \%$ of our patients. So, we recorded less Anti dsDNA percentage than they recorded in their study. Considering the other serological features, they did Anti LKM in only 3 patients and all are -ve, and by comparing that to our study it is relatively like our study as we did Anti LKM in 23 SLE patients associated with liver involvement and only one patient was +ve. We classified our 23 SLE patients associated with liver involvement into 5 major groups which were to their importance: $\mathrm{AlH}$, cholestatic jaundice, portal thrombosis followed by the $4^{\text {th }}$ which was cirrhosis then liver congestion and fatty changes in the liver while the $6^{\text {th }}$ group was viral hepatitis in the form with HCV. Considering the major groups of liver involvement in 23 SLE patients associated with liver involvement we found that Fatty changes in liver was the most common group presented in 13 patients $(56.6 \%)$ followed by both Autoimmune hepatitis $(\mathrm{AlH})$ and Viral hepatitis in the form of HCV which were presented equally in 6 patients $(26.08 \%)$. The $4^{\text {th }}$ presenting group was portal thrombosis presented in 2 patients ( $8.6 \%$ ) followed by the remaining 3 groups which had the same presentation were cholangitis, liver congestion, and liver cirrhosis which presented all in only one patient (4.3\%). And again, our results agreed with Chowdhary who found that the major groups of liver involvement in their SLE patients in order were fatty changes followed by $\mathrm{AlH}$, followed by Viral hepatitis which is like our results. We divided our 200 SLE patients into $1^{\text {st }}$ two major groups: group $A$ which includes 6 patients associated with $\mathrm{AlH}$ and group B which includes 194 patients without AlH. By comparing demographic data between group $A$ and $B$ we detected a significant relation between the mean age of onset in both groups $(p=0.034)$ while no significant relation was detected between mean ages in both groups $(p=0.130)$ and between the mean disease duration in both groups $(p=0.145)$. If compared this result with another large study did by Tojo who did their study on $\mathrm{AlH}$ in SLE. He detected that there was no significant relation between demographic data if compared between SLE patients with AlH \& SLE patients without AlH which is nearly similar to our study except that we found only significant relation between mean age of onset in both groups ${ }^{(16)}$. By comparing major organs involved in group $A \& B$, we found that there is no significant relation was found between organ involved in group $A \& B$. Then we compared the clinical presentation between group $A \& B$ which includes the elevated liver enzymes, elevated total \&direct bilirubin, elevated cholesterol, ascites, lower limb edema, abdominal tenderness, hepatomegaly and splenomegaly. We detected only a significant relation between ascites found in group $A \& B(p=0.007)$. The assessment of organ damage by using SLICC was compared between both groups $A \& B$ and reveals a significant relation between SLICC in both groups $(p=0.010)$. The $2^{\text {nd }}$ major group of liver in our studied 23 SLE patients associated with liver involvement was the cholestataic jaundice. The $3^{\text {rd }}$ major group of liver in 23 SLE patients was the portal venous thrombosis which consists of two female patients their ages were $25 \& 27$ years old, their ages of onset were 16\&20 years old and their disease duration was 9\& 7 years. We divided our 200 SLE patients into $4^{\text {th }}$ two major groups: Group C consists of 13 SLE patients having fatty changes in the liver and group D consists of 187 SLE patients without fatty changes in the liver. We compared the demographic data between both groups $C \& D$ and no significant relation was found. Then we compared the 
major organs involved in both groups and no significant relation was found between group $C \& D$ considering the major organs involved. While by comparing the clinical presentation between group $C \& D$ we found only one significant relation between lower limb edema in both groups $(p=0.007)$. The $5^{\text {th }}$ major group of liver in 23 SLE patient with liver cirrhosis Considering only one female patient had cirrhotic liver, her age was 27 years, age of onset of the disease was 20 years and her disease duration was 7 years. The cirrhotic patient showed musculoskeletal involvement in the form of arthralgia, lung involvement showing pleurisy, she clinically examined and showed hepatomegaly and lower limb edema. The $6^{\text {th }}$ major liver group in our studied 23 SLE patients associated with liver involvement was the liver congestion. A 27 years old female patient had liver congestion with a disease duration of 5 years and her age of onset was 22 years old. And finally, we classified our 200SLE patients into last 2 major groups which are group E consists of 6 SLE patients having HCV and group $F$ consists of 194 SLE patients without HCV. We compared demographic data between group $\mathrm{E}$ \& $F$ and no significant relations were found. No significant difference was found between group $E \& F$ as regards the major organs involved, the clinical presentation, and the calculated SLICC.

\section{Conclusion}

We conclude that the liver is the least involved organ in our lupus patients and that the younger the age of lupus patients the more the organ damage.

\section{References}

1. Kamen DL, and Alele JD. Skeletal manifestations of systemic autoimmune diseases. Curr Opin Endocrinol Diabetes Obes. 2010;17(6):540-5.
2. Rahman A, David A, Isenberg: "Review Article: Systemic lupus erythematosus. Engl J Med. 2008:358(9):929-939.

3. Kalifa M, Benjazia E, Rezgui $A$ et al. Lupus hepatitis: a case series of 12 patients. Rev Med Intern. (2011): 32:347-9.

4. Van Hoek B. The spectrum of liver disease in systemic lupus erythematosus. Neth $J$ Med. (1996): 48(6):244-53.

5. Matsumoto T, Yoshimine T, Shimouchi K, et al. The liver in systemic lupus erythematosus: pathologic analysis of 52 cases and review of Japanese Autopasy Registry Data. Hum Pathol. (1992): 23(10):1151-8.

6. Efe C, Purnak T, Ozaslan E, et al. Autoimmune liver disease in patients with systemic lupus erythematosus retrospective analysis of 147 cases. Scand J Gastroenterol. (2011): 46(6):732-7.

7. Arvanitaki $M$, Alder $M$. Nodular regenerative hyperplasia of the liver. A review of 14 cases. Hepatogastroenterology. (2011): 48(41): $1425-6$.

8. Kim YG, Ha HK, Nah SS, Lee CK, Moon HB, Yoo B: Acute abdominal pain in systemic lupus erythmatosus. Lupus enteritis. Ann Rheum Dis. (2002): 65(11):424-29.

9. Perlemuter $\mathrm{G}$, Chaussade $\mathrm{S}$, Wechsler $\mathrm{B}$, et al. Systemic lupus erythematosus: A companion to Rheumatology. Scand J Rheumatol. (2003): 32:191-5.

10. Zazzetti F, Buschiazzo A, Villamil FG, Barreira JC. Liver transplantation in systemic lupus erythmatosus: Case report and review of the literature. Lupus. (2011): 20(1):90-4.

11. Hochberg MC. Updating the American College of Rheumatology revised criteria for the classification of systemic lupus erythematosus. Arthritis Rheum. (1997): 40(9):1725-27.

12. William J, Berger T, Elston D. Andrews Diseases of the skin: Clinical Dermatology (10th ed.). Saunders. (2005): 10(2):2216-21.

13. Krawitt EL. Clinical features and management of autoimmune hepatitis. World J Gastroenterol (2008): 14(21):3301-5.

14. Irving KS, Sen D, Tahir H, Pilkington C, Isenberg D.: A comparison of autoimmune liver disease in juvenile and adult populations with systemic lupus erythematosus-a 
retrospective review of cases. Rheumatology. (2007): 46(7): 1171-3.

15. Chowdhary VR, Crowson CS, Poterucha JJ, Moder KG. Liver involvement in systemic lupus erythematosus: case review of 40 patients. J Rheumatol. 2008; 35 (11):215964.

16. Tojo J, Ohira H, Abe K, et al. Autoimmune hepatitis accompanied by systemic lupus erythematosus. Intern Med. (2004): 43(3):258-62. 
